\title{
Decomposition of Differential Games with Multiple Targets
}

\author{
Adriano Festa - Richard B. Vinter
}

Received: date / Accepted: date

\begin{abstract}
This paper provides a decomposition technique for the purpose of simplifying the solution of certain zero-sum differential games. The games considered terminate when the state reaches a target, which can be expressed as the union of a collection of target subsets considered as 'multiple targets'; the decomposition consists of replacing the original target by each of the target subsets. The value of the original game is then obtained as the lower envelope of the values of the collection of games resulting from the decomposition, which can be much easier to solve than the original game. Criteria are given for the validity of the decomposition. The paper includes examples, illustrating the application of the technique to pursuit/evasion games and to flow control.
\end{abstract}

Keywords Differential games · viscosity solutions · decomposition techniques.

Mathematics Subject Classification (2000) 49 N70 $\cdot 35 \mathrm{D} 40 \cdot 49 \mathrm{M} 27$

\section{Introduction}

We propose a decomposition technique for a two-player, zero-sum differential game. In this game, the state evolves according to a differential equation, which depends on the actions of the two players (the $a$-player and the $b$-player). The game terminates when the state first enters a region $\mathcal{T}$, called the target set. The pay-off, which involves a discounted running cost that depends on the state and controls up to the 'exit time', can then be evaluated. The goals of

A. Festa

RICAM - Austrian Academy of Sciences (ÖAW)

Altenberger Straße, 4040 Linz, Austria.

a.festa@ricam.oeaw.ac.at

R. B. Vinter

Imperial College - EEE Department,

Exhibition Road, SW7 2AZ London, UK.

r.vinter@imperial.ac.uk 
the $a$-player and $b$-player are to maximize and minimize the pay-off, respectively. In some cases of interest, of which we give examples, the technique leads to a significant simplification of the solution.

As usual, the value function, which we shall denote $u($.$) , is the value of the$ game, regarded as a function of the initial time and state. In this paper, attention focuses on situations in which the target $\mathcal{T}$ can be represented as the union of a finite number of closed subset $\mathcal{T}_{j}, j=1, \ldots, m$, thus $\mathcal{T}=\cup_{j=1}^{m} \mathcal{T}_{j}$. Here, the two players have a choice over which target subset to exit into.

Consider the family of $m$ 'reduced' zero-sum differential games, which is obtained by replacing the target set by each of the target subsets. Denote the value functions of the reduced games by $u_{j}(),. j=1, \ldots, m$.

Of special interest in this paper are cases in which the value functions $u_{j}($.$) ,$ $j=1, \ldots, m$, for the target subsets are easier to calculate than the value function $u($.$) for the full target \mathcal{T}$ and when $u($.$) can be constructed as the lower$ envelope of the $u_{j}($.$) 's, i.e., for each x, u(x)=\min \left\{u_{j}(x): j=1, \ldots, m\right\}$. In these cases, the problem of determining the upper value function for the given target can be 'decomposed' into a collection of simpler problems of determining the value functions for the individual target subsets.

The motivation for seeking a decomposition of this nature is as follows. Optimal control problems are special cases of differential games in which the constraint set $A$ for the $a$-player is a single point; there is then only one possible action for the $a$-player, which can therefore be effectively ignored. For optimal control problems, the decomposition is always valid, since replacing $\mathcal{T}$ by one particular $\mathcal{T}_{j}$ amounts to a strengthening of the problem constraints, and cannot therefore reduce the value. So, given any $x$, we have $u(x) \leq u_{j}(x)$ for all $j$ and therefore $u(x) \leq \min _{j} u_{j}(x)$. On the other hand, an optimal policy, for the given initial state $x$, must result in the state trajectory exiting into $\mathcal{T}_{\bar{k}}$ for some $\bar{k}$. But then $u(x) \geq u_{\bar{k}}(x) \geq \min _{j} u_{j}(x)$. These inequalities validate the decomposition.

When the presence of the $a$-player is restored and we are dealing with a true differential game, decomposition is a much more complicated issue. There are nontheless interesting cases when the decomposition, based on consideration of the target subsets, can still be achieved. The goal of this paper is to give criteria for decomposition, and to illustrate their application.

We shall assume that the value functions involved are unique viscosity solutions of the Hamilton Jacobi Isaacs (HJI) equation with appropriate boundary conditions. This means that checking the validity of the decomposition reduces to answering the question: when is the lower envelope of a family of viscosity solutions to a particular HJI equation also a viscosity solution? In Section 2 we give two criteria $((E)$ and $(C))$ under which the answer is affirmative. $(E)$ is more general, but $(C)$ is often easier to verify directly. $(C)$ is satisfied, in particular, when $F(x, u,$.$) is convex. This is a well-known fact: the viscosity so-$ lution property is preserved under the operation of taking the lower envelopes, for convex Hamiltonians. Notice that, for optimal control problems $F(x, u,$. is always convex, so this fact is consistent with the earlier observation that, for optimal control problems, regarded as special cases of differential games, 
the decomposition is possible. However $(C)$ is weaker than 'full' convexity of $F(x, u,$.$) , because it requires us to check, for each x \in \mathbb{R}^{n} \backslash \mathcal{T}$, the convexity inequality only w.r.t. gradient vectors of the minimizing $u_{j}($.$) 's at x$. In the examples we consider, this (restricted sense) convexity condition is satisfied while full convexity fails.

For the decomposition to be useful, the value functions associated with each of the target subsets must be simpler to calculate than the value function for the full target. We provide examples from pursuit/evasion games and also from flow control, to illustrate two mechanisms for simplification. Firstly, in the pursuit evasion games here considered, each of the decomposed games involves the interaction between just one pursuer and one evader and, for this reason, has a lower state dimension than the original game. Secondly, in the flow control example that follows, the simplification is that each of the decomposed problems reduces to an optimal control problem which can be solved by analysis.

The idea of patching together solutions to HJI equations on subdomains to construct a solution to the HJI equation on the full the domain satisfying the relevant boundary conditions is implicit in earlier work, examples of which appear in [15] and is implicit in numerical methods such as those described in [4]. This paper proposes a systematic approach ('decomposition of the upper value function via the lower envelope operation') to patching together different solutions, in certain cases when the target is expressed as the union of target subsets. It is worth to mention also, as the results presented here furnish the theoretical elements that permit to build the numerical technique proposed in [11].

Overcoming the 'curse of dimensionality' is a major challenge in the solution of differential games with a high state dimension. This paper provides one approach. But we emphasize that 'decomposition via target subsets' depends on the game having a rather special structure. It is justified by the interesting special cases to which it applies (several of which we analyse here). It should not be regarded as a universal approach. Indeed the assumption that the value function is continuous excludes many classical games such as the Homicidal Chauffeur Problem, characterized by a free boundary between the set of initial states from which the target can be reached, and its complement on which the value is infinite.

The term 'decomposition' is used in this paper is interpreted specifically as 'decomposition via target subsets'. 'Decomposition' is used in different senses in the differential games literature and optimal control, broadly to describe procedures involving the solution of HJI equations on subdomains. See, for example, [22], [4]. Some results of this paper were announced, without proof, in $[12]$.

Computational methods for differential games are a rather large theme of research. We refer, for the simulations shown in this paper to [9]. 


\section{Problem Formulation}

The state trajectory associated with open loop policies $a($.$) and b($.$) ('open$ loop policies' are defined below), for a specified initial state $x_{0}$, is given by the (absolutely continuous) solution of the differential equation

$$
\left\{\begin{array}{l}
\dot{x}(t)=f(x(t), a(t), b(t)), \text { a.e. } \\
x(0)=x_{0} .
\end{array}\right.
$$

Here, $f(., .,):. \mathbb{R}^{n} \times \mathbb{R}^{m_{a}} \times \mathbb{R}^{m_{b}} \rightarrow \mathbb{R}^{n}$ is a given function. Open loop policies $a($.$) and b($.$) of the two players take values in specified sets A \subset \mathbb{R}^{m_{a}}$ and $B \subset \mathbb{R}^{m_{b}}$ respectively. We write the solution $x\left(t ; x_{0}, a(),. b().\right)$. It is assumed that hypotheses are imposed on the data ensuring that a solution exists and it is unique. We also specify a closed set $\mathcal{T} \subset \mathbb{R}^{n}$ called the 'target'. The first entry time $\tau$ for $x\left(t ; x_{0}, a(),. b().\right)$ (into $\mathcal{T}$ ) is

$$
\tau:=\sup \left\{t: x\left(t^{\prime} ; x_{0}, a(.), b(.)\right) \notin \mathcal{T} \text { for all } t^{\prime} \in[0, t)\right\} .
$$

Let $\mathcal{A}$ and $\mathcal{B}$ be the spaces of open loop policies for the $a$-player and $b$-player respectively, namely

$$
\begin{aligned}
& \mathcal{A}:=\left\{a(.):\left[0, \infty\left[\rightarrow \mathbb{R}^{m_{a}}: a(.) \text { meas. and } a(t) \in A \text { a.e. }\right\},\right.\right. \\
& \mathcal{B}:=\left\{b(.):\left[0, \infty\left[\rightarrow \mathbb{R}^{m_{b}}: b(.) \text { meas. and } b(t) \in B \text { a.e. }\right\} .\right.\right.
\end{aligned}
$$

For $a(.) \in \mathcal{A}$ and $b(.) \in \mathcal{B}$ the pay-off is

$$
J\left(x_{0}, a(.), b(.)\right)=\int_{0}^{\tau} e^{-\lambda t} l\left(x\left(t ; 0, x_{0}, a(.), b(.)\right), a(t), b(t)\right) d t,
$$

in which $l(., .,):. \mathbb{R}^{n} \times \mathbb{R}^{m_{a}} \times \mathbb{R}^{m_{b}} \rightarrow \mathbb{R}$ (the payoff integrand) is a given function and $\lambda \geq 0$ (the discount factor) is a given number. Here, $\tau$ is the first entry time for $x\left(t ; x_{0}, a(),. b().\right)$.

Following Elliot-Kalton [8], we interpret 'closed loop' policies for the $a$-player and $b$-player respectively as

$$
\begin{aligned}
& \Phi:=\{\phi: \mathcal{B} \rightarrow \mathcal{A}: \phi \text { is non-anticipative }\} \\
& \Psi:=\{\psi: \mathcal{A} \rightarrow \mathcal{B}: \psi \text { is non-anticipative }\}
\end{aligned}
$$

Here, ' $\phi($.$) is non-anticipative' in the first relation means: 'for any t^{\prime} \geq 0$, and $b_{1}(),. b_{2}(.) \in \mathcal{B}$,

$$
b_{1}(t)=b_{2}(t) \text { a.e. } t \in\left[0, t^{\prime}\right] \Longrightarrow \phi\left(b_{1}(.)\right)(t)=\phi\left(b_{2}(.)\right)(t) \text { for a.e. } t \in\left[0, t^{\prime}\right] \text { '. }
$$

' $\psi($.$) is non-anticipative' in the second defining relation is analogously defined.$ Using these interpretations, we define the upper and lower values $u^{*}(x)$ and $u_{*}(x)$ of the game, for a given starting point $x \in \mathbb{R}^{n}$, to be

$$
u^{*}(x)=\sup _{\phi \in \Phi} \inf _{b \in \mathcal{B}} J\left(x(. ; x, \phi(b(.)), b(.)), \quad u_{*}(x)=\inf _{\psi \in \Psi} \sup _{a \in \mathcal{A}} J(x(. ; x, a(.), \psi(a(.))) .\right.
$$


If $u^{*}()=.u_{*}($.$) , we call this function the value function and write it u($.$) .$ Define the real valued function $F(., .,$.$) with domain in \mathbb{R}^{n} \times \mathbb{R} \times \mathbb{R}^{n}$

$$
F(x, u, p)=\lambda u+\inf _{a \in A} \sup _{b \in B}\{-p \cdot f(x, a, b)-l(x, a, b)\},
$$

There is an extensive literature providing precise conditions on the data, target, etc., which includes the Isaac's condition

$\inf _{a \in A} \sup _{b \in B}\{-p \cdot f(x, a, b)-l(x, a, b)\}=\sup _{b \in B} \inf _{a \in A}\{-p \cdot f(x, a, b)-l(x, a, b)\}$,

under which the value function $u($.$) exists and can be characterized as the$ unique continuous viscosity solution of the HJI (Hamilton Jacobi Isaacs) equation:

$$
\begin{cases}F(x, u, D u)=0 & \text { for } x \in \mathbb{R}^{n} \backslash \mathcal{T}, \\ u(x)=0 & \text { for } x \in \mathcal{T},\end{cases}
$$

in which $D u(x)$ denotes 'gradient of $u($.$) at x$ ', and when maximizing closed loop policies for the $a$-player can be obtained from knowledge of $u($.$) . See [1],$ [3], [18] for expository material on these topics, and [2] for numerical aspects. Now suppose that the target $\mathcal{T}$ can be represented as the union of a finite number of closed sets $\mathcal{T}_{j}, j=1, \ldots, m$ :

$$
\mathcal{T}=\cup_{j=1}^{m} \mathcal{T}_{j}
$$

We define $u_{j}(),. j=1, \ldots, m$ to be the value function that results when the target $\mathcal{T}$ is replaced by the subset $\mathcal{T}_{j}$, for $j=1, \ldots, m$. We shall examine cases in which the value functions $u_{j}(),. j=1, \ldots, m$, for the target subsets are easier to calculate than the value function $u($.$) for the full target \mathcal{T}$ and when $u($.$) can constructed as the lower envelope of the u_{j}($.$) 's, thus:$

$$
u(x)=\min \left\{u_{j}(x): j=1, \ldots, m\right\} .
$$

\section{Properties of the Lower Envelope of a Family of Viscosity Solutions}

Take a function $F(., .,):. \mathbb{R}^{n} \times \mathbb{R} \times \mathbb{R}^{n} \rightarrow \mathbb{R}$ and consider the partial differential equation

$$
F(x, u(x), D u(x))=0 .
$$

Definition 3.1 Take an open subset $\Omega \subset \mathbb{R}^{n}$ and a function $u():. \Omega \rightarrow \mathbb{R}$. Then $u($.$) is a continuous viscosity subsolution of (3) on \Omega$ iff it is continuous and, for each $x \in \Omega$,

$$
F(x, u(x), p) \leq 0, \quad \forall p \in D^{+} u(x) .
$$

$u($.$) is a continuous viscosity supersolution of (3) on \Omega$ iff it is continuous and, for each $x \in \Omega$,

$$
F(x, u(x), p) \geq 0, \quad \forall p \in D^{-} u(x) .
$$

$u($.$) is a continuous viscosity solution of (3) on \Omega$ iff it is both a continuous subsolution and supersolution of (3) on $\Omega$. 
Here, $D^{+} u(x)$ and $D^{-} u(x)$ denote, respectively, the Fréchet superdifferential and subdifferential of the continuous function $u($.$) defined on an open subset$ of $\mathbb{R}^{n}$ containing the point $x$ :

$$
\begin{aligned}
& D^{+} u(x):=\left\{p \in \mathbb{R}^{n}: \limsup _{y \rightarrow x} \frac{u(y)-u(x)-p \cdot(y-x)}{|x-y|} \leq 0\right\}, \\
& D^{-} u(x):=\left\{p \in \mathbb{R}^{n}: \liminf _{y \rightarrow x} \frac{u(y)-u(x)-p \cdot(y-x)}{|x-y|} \geq 0\right\} .
\end{aligned}
$$

(For the analysis of this paper it is helpful to employ a definition of continuous viscosity solutions in terms of one-sided Fréchet differentials which is equivalent to the standard definition in terms of gradients of smooth majorizing and minoring functions [6].)

The following proposition gives conditions under which the lower envelope of a collection of continuous viscosity solutions of (3) is also a continuous viscosity solution, expressed in terms of the limiting superdifferential $\partial^{L}(x)$ of the continuous function $u($.$) at x$ :

$\partial^{L} u(x):=\left\{p: \exists\right.$ sequences $p_{i} \rightarrow p$ and $x_{i} \rightarrow x$ s.t. $p_{i} \in D^{+} u\left(x_{i}\right)$ for each $\left.i\right\}$.

Proposition 3.1 Take a collection of closed sets $\mathcal{T}_{j} \subset \mathbb{R}^{n}, j=1, \ldots, m$. For each $j$, let $u_{j}($.$) be a scalar valued function with domain \mathbb{R}^{n} \backslash \mathcal{T}_{j}$. Define

$$
I(x)=\left\{j \in\{1, \ldots, m\}: u_{j}(x)=\min _{j^{\prime}} u_{j^{\prime}}(x)\right\} \quad \text { for each } x \in \mathbb{R}^{n} \backslash \cup_{j=1}^{m} \mathcal{T}_{j}
$$

and $\Sigma=\left\{x \in \mathbb{R}^{n} \backslash \cup_{j=1}^{m} \mathcal{T}_{j}:\right.$ Cardinality $\left.\{I(x)\}>1\right\}$.

Take $\bar{u}():. \mathbb{R}^{n} \backslash \cup_{j=1}^{m} \mathcal{T}_{j} \rightarrow \mathbb{R}$ to be the lower envelope

$$
\bar{u}(x)=\min _{j}\left\{u_{j}(x)\right\} .
$$

Consider the following hypotheses:

(E): for any $x \in \Sigma$, any set of vectors $\left\{p_{j}: j \in I(x)\right\}$ such that $p_{j} \in \partial^{L} u_{j}(x)$ for each $j \in I(x)$, and any convex combination $\left\{\lambda_{j}: j \in I(x)\right\}$,

$$
F\left(x, \bar{u}(x), \sum_{j \in I(x)} \lambda_{j} p_{j}\right) \leq 0 .
$$

$(C)$ : for any $x \in \Sigma$, any set of vectors $\left\{p_{j}: j \in I(x)\right\}$ such that $p_{j} \in \partial^{L} u_{j}(x)$ for each $j \in I(x)$, and any convex combination $\left\{\lambda_{j}: j \in I(x)\right\}$,

$$
F\left(x, \bar{u}(x), \sum_{j \in I(x)} \lambda_{j} p_{j}\right) \leq \sum_{j \in I(x)} \lambda_{j} F\left(x, \bar{u}(x), p_{j}\right),
$$

We have:

(a): Suppose that, for each $j, u_{j}($.$) is a continuous viscosity supersolution of$ (3) on $\mathbb{R}^{n} \backslash \mathcal{T}_{j}$. Then $\bar{u}($.$) is a continuous viscosity supersolution of (3) on$ $\mathbb{R}^{n} \backslash \cup_{j=1}^{m} \mathcal{T}_{j}$. 
(b): Suppose that, for each $j, u_{j}($.$) is a continuous viscosity subsolution of (3)$ on $\mathbb{R}^{n} \backslash \mathcal{T}_{j}$. Suppose also that $F(., .,$.$) is continuous and, for each x \in \Sigma$ and $j \in I(x), u_{j}($.$) is Lipschitz continuous on a neighbourhood of x$.

We have:

(i): $(E) \Longrightarrow \bar{u}($.$) is a continuous viscosity subsolution of (3)$ on $\mathbb{R}^{n} \backslash \cup_{j=1}^{m} \mathcal{T}_{j}$.

(ii): If, additionally, we assume that for each $x \in \Sigma$ and $j \in I(x), u_{j}($.$) is C^{1}$ on a neighbourhood of $x$, then $\bar{u}($.$) is a continuous viscosity subsolution$ of (3) on $\mathbb{R}^{n} \backslash \cup_{j=1}^{m} \mathcal{T}_{j} \Longrightarrow(E)$.

(iii): $(C) \Longrightarrow(E)$.

\section{Remarks}

$1)$ : The proof of the proposition is based on well-known estimates for onesided differentials to lower envelope functions, in terms of the one-sided differentials to the constituent functions (the 'Max Rule'). Such estimates are studied in depth in [19].

2): The proposition treats separately (in parts (a) and (b)) the preservation of the supersolution and subsolution properties of viscosity solutions under the operation of taking the lower envelope, because much weaker hypotheses need be imposed in connection with supersolutions.

3): Assertions b(i) and b(iii) provide two sufficient conditions for the lower envelope of a family of continuous viscosity subsolutions also to be a continuous viscosity subsolution when $F(, ., .,$.$) is continuous and the u_{j}$ 's are Lipschitz continuous near $\Sigma$, namely $(E)$ and $(C) .(C)$ is a more restrictive hypothesis, but it is useful because, as illustrated in the following examples, it can be easier to verify directly.

4): Assertion b(ii) suggests that the sufficient condition in b(i) is close to optimal since, when the $u_{j}($.$) 's are C^{1}$ near $\Sigma$, condition (E) is actually necessary for the lower envelope to be a continuous viscosity subsolution of (3). This necessary condition will be used to reject a candidate for the upper value function in an example below.

(5): The proposition is an analytical tool for decomposing a differential game (associated with the value function $\bar{u}($.$) ) into a collection of simpler prob-$ lems. The critical hypothesis in this proposition is $(E)$ (or $(C)) .(C)$ is automatically satisfied when $F(x, u,$.$) is convex. This special case of the$ proposition is well-known [6]. However $(C)$ imposes a convexity type condition on $F(x, u,$.$) , only with respect to selected vectors in its domain. In$ some cases, examples of which given below, the restricted sense convexity hypothesis is satisfied but the full convexity hypothesis is violated.

Proof of Prop. 3.1

(a): Suppose that $u_{j}($.$) is a continuous viscosity supersolution of (3) on \mathbb{R}^{n} \backslash \mathcal{T}_{j}$ for each $j$. Take any $x \in \mathbb{R}^{n} \backslash \cup_{j=1}^{m} \mathcal{T}_{j}$ and $p \in D^{-} \bar{u}(x)$. Then

$$
\bar{u}\left(x^{\prime}\right)-\bar{u}(x) \geq p \cdot\left(x^{\prime}-x\right)-o\left(\left|x^{\prime}-x\right|\right),
$$

for all $x^{\prime} \in \mathbb{R}^{n} \backslash\left(\cup_{j=1}^{m} \mathcal{T}_{j}\right)$, lying in some neighborbood of $x$.

(Here, $o():. \mathbb{R}^{+} \rightarrow \mathbb{R}^{+}$is some function such that $\lim _{s \downarrow 0} o(s) / s \rightarrow 0$.) 
Choose any $j \in I(x)$. We know that $u_{j}(x)=\bar{u}(x)$ and $u_{j}\left(x^{\prime}\right) \geq \bar{u}\left(x^{\prime}\right)$. It follows that, for all $x^{\prime} \in \mathbb{R}^{n} \backslash\left(\cup_{j=1}^{m} \mathcal{T}_{j}\right)$,

$$
u_{j}\left(x^{\prime}\right)-u_{j}(x) \geq p \cdot\left(x^{\prime}-x\right)-o\left(\left|x^{\prime}-x\right|\right) .
$$

But then $p \in D^{-} u_{j}(x)$ and, since $u_{j}($.$) is a continuous viscosity supersolution,$ we have $F\left(x, u_{j}(x), p\right) \geq 0$. It follows that $F(x, \bar{u}(x), p) \geq 0$. Since $\bar{u}($.$) is con-$ tinuous, we have established that $\bar{u}($.$) is a continuous viscosity supersolution$ of $(3)$ on $\mathbb{R}^{n} \backslash\left(\cup_{j=1}^{m} \mathcal{T}_{j}\right)$.

(b)(i): Suppose that $u_{j}($.$) is a continuous viscosity subsolution of (3) on \mathbb{R}^{n} \backslash \mathcal{T}_{j}$ for each $j$. Take any $x \in \mathbb{R}^{n} \backslash\left(\cup_{j=1}^{m} \mathcal{T}_{j}\right)$ and $p \in D^{+} \bar{u}(x)$. We must show that

$$
F(x, \bar{u}(x), p) \leq 0 .
$$

Suppose first that $x \notin \Sigma$, i.e. $I(x)$ contains a single index value $j$. Then, since the $u_{i}($.$) 's are continuous, \bar{u}\left(x^{\prime}\right)=u_{j}\left(x^{\prime}\right)$ for all $x^{\prime}$ in some neighbourhood of $x$. It follows that $p \in D^{+} u_{j}$ and so $F\left(x, u_{j}(x), p\right)(=F(x, \bar{u}(x), p)) \leq 0$. We have confirmed (4) in this case.

It may be assumed then that $x \in \Sigma$. Now, $u_{j}($.$) is Lipschitz continuous on a$ neighbourhood of $x$ for each $j \in I(x)$. Since $p \in D^{+} \bar{u}(x)$, it is certainly the case that $p \in \partial^{L} \bar{u}(x)$. Using that $\bar{u}\left(x^{\prime}\right)$ coincides with $\min \left\{u_{j}\left(x^{\prime}\right): j \in I\left(x^{\prime}\right)\right\}$ for $x^{\prime}$ in some neighbourhood of $x$, we deduce from the Max Rule for limiting subdifferentials of Lipschitz continuous functions (see, e.g., [24, Thm. 5.5.2]) applied to $-\bar{u}($.$) the following representation for p$ :

$$
p=\sum_{j \in I(x)} \lambda_{j} p_{j},
$$

for some convex combination $\left\{\lambda_{j}: j \in I(x)\right\}$ and vectors $p_{j} \in \partial^{L} u_{j}(x)$, $j \in I(x)$. But then, by hypothesis (E),

$$
F(x, \bar{u}(x), p)=F\left(x, \bar{u}(x), \sum_{j \in I(x)} \lambda_{j} p_{j}\right) \leq 0 .
$$

We have confirmed (4) and so (b)(i) is true.

(b)(ii): Take any $x \in \Sigma$. Suppose that the $u_{j}$ 's are continuously differentiable on a neighbourhood of $x$ and that $\bar{u}($.$) is a viscosity solution. Take any convex$ combination $\left\{\lambda_{i}\right\}$ on $I(x)$. Then, for all $x^{\prime}$ in some neighborhood of $x$,

$\bar{u}\left(x^{\prime}\right)-\bar{u}(x) \leq \sum_{i \in I(x)} \lambda_{i}\left(u_{i}\left(x^{\prime}\right)-u_{i}(x)\right) \leq \sum_{i \in I(x)} \lambda_{i} D u_{i}(x) \cdot\left(x^{\prime}-x\right)+o\left(\left|x^{\prime}-x\right|\right)$.

This last inequality tells us that $\sum_{i \in I(x)} \lambda_{i} D u_{i}(x)$ is a member of the Frechet superdifferential of $\bar{u}($.$) at x$. But then, since $\bar{u}($.$) is a viscosity subsolution,$

$$
F\left(x, \bar{u}(x), \sum_{j \in I(x)} \lambda_{j} p_{j}\right) \leq 0 .
$$

We have confirmed that $(E)$ is true. 
(b)(iii): Take any convex combination $\left\{\lambda_{j}\right\}$ on $I(x)$ and vectors $p_{j} \in \partial^{L} u_{i}(x)$ for $j \in I(x)$. It follows from the definition of the limiting superdifferential that, for each $j$, there exist sequences $x_{j}^{i} \rightarrow x$ and $p_{j}^{i} \rightarrow p_{j}$ such that $p_{j}^{i} \in D^{+} u_{i}\left(x_{j}^{i}\right)$ for $i=1,2, \ldots$ But then, for each $j \in I(x)$,

$$
F\left(x_{j}^{i}, u_{j}\left(x_{j}^{i}\right), p_{j}^{i}\right) \leq 0, i=1,2, \ldots,
$$

since the $u_{j}($.$) 's are viscosity subsolutions. It follows that$

$$
\sum_{j \in I(x)} \lambda_{j} F\left(x_{j}^{i}, u^{j}\left(x_{j}^{i}\right), p_{j}^{i}\right) \leq 0, i=1,2, \ldots
$$

Noting the continuity of $F(.,, .$,$) and also the u_{j}($.$) 's, we may pass to the limit$ as $i \rightarrow \infty$ to obtain

$$
\sum_{j \in I(x)} \lambda_{j} F\left(x, u_{j}(x), p_{j}\right) \leq 0
$$

Assume $(C)$. Then

$$
F\left(x, \bar{u}(x), \sum_{j \in I(x)} \lambda_{j} p_{j}\right) \leq \sum_{j \in I(x)} \lambda_{j} F\left(x, u_{j}, p_{j}\right) \leq 0,
$$

which is $(E)$.

\section{Pursuit Evasion Games}

Pursuer/evader games are examples of the game posed in the Introduction. There is an extensive literature on such games, going back to Rufus Isaacs' work in the 1960's, and his monograph [15] contains many examples. Expository material is to be found in [13], [18]. We note also [7], [16], [14], [16], [21], and [23]. But none of these references systematically address decompositions of the game, each element of which is generated by a target subset. Pursuer/evader games is an application area for the methods proposed in this paper; they provide exemplar problems, both where decomposition is possible, and where it is not.

We consider zero sum differential games which terminate when one of the pursuers is sufficiently close to one of the evaders, where 'closeness' is understood in the sense of a specified target. The pay-off is the time until the target is attained. We analyse a number of examples, involving different numbers of pursuers and evaders, and different targets.

The $a$-player is the collection of $m_{1}$ evaders, labelled $1, \ldots, m_{1}$, and the $b$ player the collection of $m_{2}$ pursuers, labelled $m_{1}+1, \ldots, m_{1}+m_{2}$. The states of individual pursuers and evaders $x_{1}, \ldots, x_{m_{1}}$ and $x_{m+1} \ldots, x_{m_{1}+m_{2}}$ are governed by the equations

$$
\begin{aligned}
& \frac{d x_{1}}{d t}=f_{1}\left(x_{1}, a_{1}\right), \ldots, \frac{d x_{m_{1}}}{d t}=f_{m_{1}}\left(x_{m_{1}}, a_{m_{1}}\right) \\
& \frac{d x_{m_{1}+1}}{d t}=f_{m_{1}+1}\left(x_{m_{1}+1}, b_{1}\right), \ldots, \frac{d x_{m_{1}+m_{2}}}{d t}=f_{m_{1}+m_{2}}\left(x_{m_{1}+m_{2}}, b_{m_{2}}\right) .
\end{aligned}
$$


The variables $a_{1}, \ldots, a_{m_{1}}$ and $b_{1}, \ldots, b_{m_{2}}$ are interpreted as controls for the evaders and the pursuers, respectively, which are subject to the constraints

$$
a_{i} \in A_{i}, i=1, \ldots, m_{1} \text {, and } b_{i} \in B_{i}, i=1, \ldots, m_{2} .
$$

Here, $f_{i}(.,):. \mathbb{R}^{n_{i}} \times \mathbb{R}^{r_{i}} \rightarrow \mathbb{R}^{n}, i=1, \ldots, m_{1}+m_{2}$ are given functions, and $A_{i} \subset \mathbb{R}^{r_{i}}, 1, \ldots, m_{1}$ and $B_{i} \subset \mathbb{R}^{r_{i+m_{1}}}$, for $i=1, \ldots, m_{2}$, are given subsets.

We regard $a_{1}, \ldots, a_{m_{1}}$ and $b_{1}, \ldots, b_{m_{2}}$ as block components of a single evader control and pursuer control respectively.

Take the state to be $x=\operatorname{col}\left\{x_{1} \ldots, x_{m_{1}+m_{2}}\right\}$. The open loop policy spaces for evader and pursuer are

$$
\begin{aligned}
\mathcal{A}:=\left\{\text { meas. mappings } a_{i}:\left[0, \infty\left[\rightarrow \mathbb{R}^{r_{i}}, i=1, \ldots, m_{1}:\right.\right.\right. & \\
& \left.a_{i}(t) \in A_{i} \text { a.e. for each } i\right\}, \\
\mathcal{B}:=\left\{\text { meas. mappings } b_{i}:\left[0, \infty\left[\rightarrow \mathbb{R}^{r_{i+m_{1}}},\right.\right.\right. & i=1, \ldots, m_{2}: \\
& \left.b_{i}(t) \in B_{i} \text { a.e. for each } i\right\} .
\end{aligned}
$$

Write $\Phi$ for the space of non-anticipative mappings $\phi: \mathcal{B} \rightarrow \mathcal{A}$. The target set $\mathcal{T}$ is a given subset of $R^{n}$, where $n:=n_{1}+\ldots, n_{m_{1}+m_{2}}$.

The game fits the formulation of Section 1 , with $\lambda=0$ and $l(., . .)=$.1 , and is summarized as:

$$
\left(P^{\prime}\right)\left\{\begin{array}{c}
\underset{\phi \in \Phi}{\operatorname{maximize}} \underset{\left\{b_{i}\right\} \in \mathcal{B}}{\operatorname{minimize}} \int_{0}^{\tau} 1 d t \\
\left\{\begin{array}{l}
\dot{x}_{1}(t)=f_{1}\left(x_{1}(t), a_{1}(t)\right) \\
\ldots \\
\dot{x}_{m_{1}+m_{2}}(t)=f_{m_{1}+m_{2}}\left(x_{m_{1}+m_{2}}(t), b_{m_{2}}(t)\right), \text { a.e. } \\
\left(a_{1}(t), \ldots, a_{m_{1}}, b_{1}(t), \ldots, b_{m_{2}}(t)\right) \\
\quad \in A_{1} \times \ldots \times A_{m_{1}} \times B_{1} \times \ldots \times B_{m_{2}}, \text { a.e. } \\
\text { in which }\left(a_{1}(.), \ldots, a_{m_{1}}(.)\right)=\phi\left(b_{1}(.), \ldots, b_{m_{2}}(.)\right) \\
\text { and } \tau \text { is first entry time into } \mathcal{T} \\
\left(x_{1}(0), \ldots, x_{m_{1}+m_{2}}(0)\right)=\left(\bar{x}_{1}, \ldots, \bar{x}_{m_{1}+m_{2}}\right)
\end{array}\right.
\end{array}\right.
$$

for some given $\left(\bar{x}_{1}, \ldots, \bar{x}_{m_{1}+m_{2}}\right) \in \mathbb{R}^{n_{1}} \times \ldots \times \mathbb{R}^{n_{\left(m_{1}+m_{2}\right)}}$. Here $\mathcal{T}$ is a given closed subset of $\mathbb{R}^{n} \times \ldots \times \mathbb{R}^{n}$. The HJI equation is

$$
F\left(x_{1}, \ldots, x_{m_{1}+m_{2}}, D_{x_{1}} u, \ldots, D_{x_{m_{1}+m_{2}}} u\right)=0,
$$

in which

$$
\begin{gathered}
F\left(x_{1}, \ldots, x_{m_{1}+m_{2}}, p_{1}, \ldots, p_{m_{1}+m_{2}}\right)=-\sum_{i=1}^{m_{1}} H^{i}\left(x_{i}, p_{i}\right)+\sum_{i=m_{1}+1}^{m_{1}+m_{2}} H^{i}\left(x_{i},-p_{i}\right)-1 . \\
\text { Here } H^{i}\left(x_{i}, p_{i}\right):= \begin{cases}\sup _{a_{i} \in A_{i}} p_{i} \cdot f\left(x_{i}, a_{i}\right) & \text { for } i=1, \ldots m_{1} \\
\sup _{i-m_{1} \in B_{i-m_{1}}} p_{i} \cdot f\left(x_{i}, b_{i-m_{1}}\right) & \text { for } i=m_{1}+1, \ldots m_{1}+m_{2} .\end{cases}
\end{gathered}
$$

We observe that the Isaacs' condition (1) is satisfied by this class of games. 


\subsection{A Single Pursuer/Multiple Evaders Game}

Consider first a case of the pursuit/evasion game, written $\left(P^{1}\right)$, in which we have $m_{1}(=: m)>1, m_{2}=1$ and $n=1$ (a single pursuer/multiple evaders game in $1 \mathrm{D}$ space). The states of the $m$ evaders, labeled $1, \ldots, m$ and of the one pursuer, labeled $m+1$, are interpreted as the positions of the evaders and pursuer. The game terminates when the pursuer is first at a distance $r$ from one of the evaders, where $r \geq 0$ is a given constant. Accordingly, we take

$$
\mathcal{T}=\mathcal{T}_{1} \cup \ldots \cup \mathcal{T}_{m}
$$

in which, for $i=1, \ldots, m, \mathcal{T}_{i}:=\left\{\left(x_{1}, \ldots, x_{m+1}\right):\left|x_{m+1}-x_{i}\right| \leq r\right\}$.

The Hamilton-Jacobi-Isaacs equation is

$$
F^{1}\left(x_{1}, \ldots, x_{m+1}, D_{x_{1}} u, \ldots, D_{x_{m+1}} u\right)=0 \text {, }
$$

in which

$$
F^{1}\left(x_{1}, \ldots, x_{m+1}, p_{1}, \ldots, p_{m+1}\right)=-\sum_{i=1}^{m} H^{i}\left(x_{i}, p_{i}\right)+H^{m+1}\left(x_{m+1},-p_{m+1}\right)-1,
$$

where

$$
\begin{aligned}
& H^{i}\left(x_{i}, p_{i}\right):=\sup _{a_{i} \in A_{i}} p_{i} \cdot f\left(x_{i}, a_{i}\right), \quad i=1, \ldots m, \\
& H^{m+1}\left(x_{m+1},-p_{m+1}\right):=\sup _{b_{1} \in B_{1}}\left(-p_{m+1}\right) \cdot f\left(x_{m+1}, b_{1}\right) .
\end{aligned}
$$

Now take $\left(P_{i}^{1}\right)$ to be the modification of $\left(P^{1}\right)$, when $\mathcal{T}_{i}$ replaces $\mathcal{T}, i=1, \ldots, m$. Let us assume that, for each $i$, the value function $u_{i}($.$) for \left(P_{i}^{1}\right)$ is a continuous viscosity solution of (5). The following proposition tells us that we can construct a viscosity solution to $(6)$ from the $u_{i}($.$) 's, by taking the pointwise$ infimum.

Proposition 4.1 Let $u_{i}($.$) be the value function for \left(P_{i}^{1}\right)$, for $i=1, \ldots m$. Assume

(a): For $i=1, \ldots m, u_{i}($.$) is a continuous viscosity solution of (5)$ on $\mathbb{R}^{m+1} \backslash \mathcal{T}_{i}$.

(b): For any $i, j \in\{1, \ldots, m\}, i \neq j$, and $\left(x_{1}, \ldots, x_{m+1}\right) \in \mathbb{R}^{m+1} \backslash \mathcal{T}$ such that $u_{i}\left(x_{1}, \ldots, x_{m+1}\right)=u_{j}\left(x_{1}, \ldots, x_{m+1}\right), u_{i}($.$) and u_{j}($.$) are Lipschitz$ continuous on a neighborhood of $\left(x_{1}, \ldots, x_{m+1}\right)$.

Then

$$
\bar{u}\left(x_{1}, \ldots, x_{m+1}\right):=\min \left\{u_{1}\left(x_{1}, \ldots x_{m+1}\right), \ldots, u_{m+1}\left(x_{1}, \ldots, x_{m+1}\right)\right\}
$$

is a continuous viscosity solution of $(5)$ on $(\mathbb{R} \times \ldots \mathbb{R}) \backslash \mathcal{T}$. 
Remark Suppose hypotheses are imposed, ensuring that (1): for each $i$, the HJI equation for $\left(P_{i}^{1}\right)$ has a continuous viscosity solution on $\left(\mathbb{R}^{n} \times \ldots \mathbb{R}^{n}\right) \backslash \mathcal{T}_{i}$ with a continuous extension to $\mathcal{T}_{i}$, on which set the solution vanishes, and (2): the value function $\left(P^{1}\right)$ is the unique continuous viscosity solution on $\left(\mathbb{R}^{n} \times \ldots \mathbb{R}^{n}\right) \backslash \mathcal{T}$ that has a continuous extension to $\mathcal{T}_{i}$, on which set the solution vanishes. The proposition tells us that, under these circumstances, the value function $u($.$) for \left(P^{1}\right)$ can be calculated as the lower envelope of the

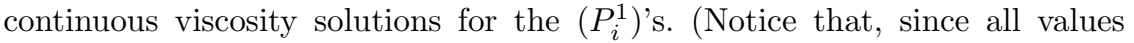
functions concerned are non-negative, and each $u_{i}($.$) is assumed to have a$ continuous extension to $\mathcal{T}_{i}$, on which set it vanishes, the lower envelope has a continuous extension to $\mathcal{T}$, on which set it vanishes.)

Proof of Prop. 4.1 Note that, for any $i, u_{i}\left(x_{1}, \ldots, x_{m+1}\right)$ depends only on the two variables $\left(x_{i}, x_{m+1}\right)$. This is because the first entry time into $\mathcal{T}_{i}$ only concerns the state trajectories associated the $i$ 'th evader and the pursuer (labelled as $m+1)$.

In view of the hypotheses imposed on the $u_{i}($.$) 's, the fact that \bar{u}($.$) is a viscosity$ solution of (5) will follow from Prop. 3.1, if we can confirm hypothesis (C) of this proposition. Take any $z=\left(x_{1}, \ldots, x_{m+1}\right) \in \mathbb{R}^{m+1} \backslash \mathcal{T}$, any index set $I(z)$ (of cardinality $l>1$ ) such that the values $u_{i}(z), i \in I(z)$, coincide, and any convex combination $\left\{\lambda_{i}\right\}$ from $I(z)$. To simplify, assume index values have been re-ordered so that $I(z)=\{1, \ldots,, l\}$. Take also $\tilde{p}^{i} \in \mathbb{R}^{n}, i=1, \ldots, l$ such that

$$
\tilde{p}^{i}:=\left(0, \ldots, 0, p_{i}^{i}, 0, \ldots, 0, p_{m+1}^{i}\right) \in \partial^{L} u_{i}(z) .
$$

(The possibly non-zero components $p_{i}^{i}$ and $p_{m+1}^{i}$ of $\tilde{p}^{i}$ appear at the $i$ 'th and $(m+1)^{\prime}$ 'th locations. We must check that $\eta\left(\lambda_{1}, \ldots, \lambda_{l}\right) \geq 0$, where

$$
\eta\left(\lambda_{1}, \ldots, \lambda_{l}\right):=\sum_{i=1}^{l} \lambda_{i} F\left(z, \tilde{p}_{i}\right)-F\left(z, \sum_{i=1}^{l} \lambda_{i} \tilde{p}_{i}\right) .
$$

Noting the special structure $(7)$ of the $\tilde{p}_{i}$ 's and the fact that $H^{i}\left(x_{i}, p_{i}\right)=0$ when $p_{1}=0$, for each $i$, we see that

$$
\begin{aligned}
\eta\left(\lambda_{1}, \ldots, \lambda_{l}\right)= & \sum_{i=1}^{l} \lambda_{i}\left(H^{m+1}\left(x_{m+1},-p_{m+1}^{i}\right)-H^{i}\left(x_{i}, p_{i}^{i}\right)\right) \\
& \left.-\left(H^{m+1}\left(x_{m+1},-\sum_{i=1}^{l} \lambda_{i} p_{m+1}^{i}\right)-\sum_{i=1}^{l} H^{i}\left(x_{i}, \lambda_{i} p_{i}^{i}\right)\right)\right) .
\end{aligned}
$$

We achieve a further simplification from the fact that $H^{i}\left(x_{i},.\right)$ is positively homogeneous, so $H^{i}\left(x_{i}, \lambda_{i} p_{m+1}^{i}\right)=\lambda_{i} H^{i}\left(x_{i}, p_{m+1}^{i}\right)$. This gives

$\eta\left(\lambda_{1}, \ldots, \lambda_{l}\right)=\sum_{i=1}^{l} \lambda_{i} H^{m+1}\left(x_{m+1},-p_{m+1}^{1}\right)-H^{m+1}\left(x_{m+1},-\sum_{i=1}^{l} \lambda_{i} p_{m+1}^{i}\right)$.

But then $\eta\left(\lambda_{1}, \ldots, \lambda_{l}\right)$ is non-negative, because the term $H^{m+1}\left(x_{m+1},.\right)$, defined by (4), is convex. The proof is complete. 


\subsection{A Multiple Pursuers/Single Evader Game}

Consider next a case of the pursuit/evasion game, written $\left(P^{2}\right)$, in which we have $m_{1}=1, m_{2}(=: m) \geq 1$ and $n=2$ (single pursuer/multiple evaders). The dynamic behavior of each player is modelled as a thrust acting on a mass, in 1D space, with saturating damping. The state equations, governing the position and velocity of each player, are taken to be, for $i=2, \ldots, m+1$,

$$
\left[\begin{array}{c}
\dot{x}_{1}^{1} \\
\dot{x}_{2}^{1}
\end{array}\right]=\left[\begin{array}{c}
x_{2}^{1} \\
-d_{1}\left(x_{2}^{i}\right)+a_{1}
\end{array}\right] \text { and }\left[\begin{array}{c}
\dot{x}_{1}^{i} \\
\dot{x}_{2}^{i}
\end{array}\right]=\left[\begin{array}{c}
x_{2}^{i} \\
-d_{i}\left(x_{2}^{i}\right)+b_{i-1}
\end{array}\right] \text {. }
$$

Here, $d_{i}():. \mathbb{R} \rightarrow \mathbb{R}, i=1, \ldots, m+1$ are given functions satisfying

$$
\left|d_{i}(y)-d_{i}\left(y^{\prime}\right)\right| \leq k_{d}\left|y-y^{\prime}\right|, \quad d_{i}(y) \leq c_{d},
$$

for all $y, y^{\prime} \in \mathbb{R}$ and $i=1, \ldots, m+1$ for some constants $k_{d}>0$ and $c_{d}>0$. The control actions the players are required to satisfy

$$
|a| \leq \alpha \text { and }\left|b_{i}\right| \leq \beta_{i} \text { for } i=1, \ldots m .
$$

for positive constants $\alpha, \beta_{1}, \ldots, \beta_{m}$. We assume that

$$
\beta_{i}>\alpha+2 \times c_{d} \quad \text { for } \mathrm{i}=1, \ldots, \mathrm{m} .
$$

The game terminates when one of the pursuers overtakes the evader. Thus, we take the target to be

$$
\mathcal{T}=\mathcal{T}_{1} \cup \ldots \cup \mathcal{T}_{m}
$$

in which, for $i=1, \ldots, m$,

$$
\mathcal{T}_{i}:=\left\{\left(x^{1}=\left(x_{1}^{1}, x_{2}^{1}\right), \ldots, x^{m+1}=\left(x_{1}^{m+1}, x_{2}^{m+1}\right): x_{1}^{i} \geq x_{1}^{1}\right\} .\right.
$$

The HJI equation is

$$
F^{2}\left(x^{1}, \ldots, x^{m+1}, D_{x^{1}} u, \ldots, D_{x^{m+1}} u\right)=0,
$$

in which

$$
\begin{aligned}
& F^{2}\left(x^{1}, \ldots, x^{m+1}, p^{1}, \ldots, p^{m+1}\right)= \\
& \left(\sum_{i=1}^{m+1}\left(-p_{1}^{i} x_{2}^{i}-p_{2}^{i} d\left(x_{2}^{i}\right)\right)\right)-\alpha \times\left|p_{2}^{1}\right|+\sum_{i=2}^{m+1}\left(\beta_{i} \times\left|p_{2}^{i}\right|\right)-1 .
\end{aligned}
$$

Let $\left(P_{i}^{2}\right)$ to be the modification of $\left(P^{2}\right)$, when the target $\mathcal{T}_{i}$ replaces $\mathcal{T}$ for $i=2, \ldots, m+1$.

Proposition 4.2 Let $u_{i}($.$) be the value function for \left(P_{i}^{2}\right)$, for $i=2, \ldots m+1$. Assume

(a): For $i=2, \ldots m+1, u_{i}($.$) is a continuous viscosity solution of (5) on$ $\left(\mathbb{R}^{2}\right)^{m+1} \backslash \mathcal{T}_{i}$ 
(b): For any $i, j \in\{2, \ldots, m+1\}, i \neq j$, and $\left(x^{1}, \ldots, x^{m+1}\right) \in\left(\mathbb{R}^{2}\right)^{m+1} \backslash \mathcal{T}$ such that

$u_{i}\left(x^{1}, \ldots, x^{m+1}\right)=u_{j}\left(x^{1}, \ldots, x^{m+1}\right), u_{i}($.$) and u_{j}($.$) are Lipschitz contin-$ uous on a neighborhood of $\left(x^{1}, \ldots, x^{m+1}\right)$.

Then

$$
\bar{u}\left(x^{1}, \ldots, x^{m+1}\right):=\min \left\{u_{1}\left(x^{1}, \ldots x^{m+1}\right), \ldots, u^{m+1}\left(x_{1}, \ldots, x^{m+1}\right)\right\}
$$

is a continuous viscosity solution of $(10)$ on $\left(\mathbb{R}^{2}\right)^{m+1} \backslash \mathcal{T}$.

Remark When, for each $i$, the HJI equation for $\left(P_{i}^{2}\right)$ has a continuous viscosity solution $u_{i}($.$) on \left(\mathbb{R}^{2}\right)^{m+1} \backslash \mathcal{T}_{i}$ (with a continuous extension to the boundary, on which it vanishes) and the value function $u($.$) for \left(P^{2}\right)$ is the unique continuous viscosity solution on $\left(\mathbb{R}^{2}\right)^{m+1} \backslash \mathcal{T}$ (with boundary values as above), the proposition provides a representation of the upper value function for $\left(P^{2}\right)$ can be obtained, as the pointwise infimum of the $u_{i}($.$) 's.$

Proof Note that, for $i=2, \ldots, m+1, u_{i}\left(x^{1}, \ldots, x^{m+1}\right)$ depends only on the two variables $\left(x^{1}, x^{i}\right)$, since the first entry time into $\mathcal{T}_{i}$ only concerns the state trajectories associated with the $i$ th pursuer and the evader. We write $u_{i}\left(x^{1}, x^{i}\right)$, suppressing irrelevant arguments in the notation. Note that assumptions (8) and (9) (which tell us that all pursuers can accelerate at a faster rate than the evader), ensure that $u_{i}\left(x^{1}, x^{i}\right)$ is finite when $x_{1}^{1} \geq x_{1}^{i}$.

The left side of the HJI equation $F^{2}=0$ can be decomposed as

$$
F^{2}=F^{21}+F^{22}-1,
$$

where $F^{21}$ and $F^{22}$, evaluated at $\left(\left(x_{1}^{1}, x_{2}^{1}\right), \ldots,\left(x_{1}^{m+1}, x_{2}^{m+1}\right),\left(p_{1}^{1}, p_{2}^{1}\right), \ldots\right.$, $\left.\left(p_{1}^{m+1}, p_{2}^{m+1}\right)\right)$, are:

$$
F^{21}=-\sum_{i=1}^{m+1}\left(p_{1}^{i} x_{2}^{i}-p_{2}^{i} d\left(x_{2}^{i}\right)\right)
$$

and

$$
F^{22}=-\alpha \times\left|p_{2}^{1}\right|+\sum_{i=2}^{m+1} \beta_{i} \times\left|p_{2}^{i}\right| .
$$

We make the following assertions, whose verification is given at the end of the proof:

Claim: Let $\bar{a}($.$) be the open loop strategy \bar{a}(.) \equiv+1$ for the evader, and let $a($. be any other open loop strategy. Take initial states for the evader $z=\left(z_{1}, z_{2}\right)$ and $z^{\prime}=\left(z_{1}^{\prime}, z_{2}^{\prime}\right)$ such that $z_{1}^{\prime} \geq z_{1}$ and $z_{2}^{\prime} \geq z_{2}$. Then

$$
x_{1}^{1}\left(t ; \bar{a}(.), z^{\prime}\right) \geq x_{1}^{1}(t ; a(.), z) \quad \text { for all } t \geq 0,
$$

where $t \rightarrow\left(x_{1}^{1}, x_{2}^{1}\right)(t ; a(), z$.$) is the state trajectory for the evader, under the$ open loop strategy $a($.$) and for initial state z$. 
(These assertions will imply positivity properties of critical components of the limiting subgradients of the $u_{i}($.$) 's (see (4.2) below), as required for application$ of the decomposition criteria of Prop. 3.1, in this example.)

Fix $i$, and consider $\left(P_{i}^{2}\right)$. We deduce from the lemma that the optimal closed loop strategy for the $a$-player (the evader) is $\bar{\phi}\left(b_{i}().\right) \equiv+1$, for arbitrary initial state $\left(x^{1}=\left(x_{1}^{1}, x_{2}^{1}\right), x_{1}^{i}=\left(x_{1}^{i}, x_{2}^{i}\right)\right)$ such that $x_{1}^{1}>x_{1}^{i}$. Furthermore, if the $a$ player applies this optimal strategy then, for any open loop strategy $b_{i}($.$) , the$ effect of increasing the $x_{2}^{1}$ component of the initial state is to increase the first interception time. We conclude that

$$
x_{2}^{1} \rightarrow u_{i}\left(\left(x_{1}^{1}, x_{2}^{1}\right),\left(x_{1}^{i}, x_{2}^{i}\right)\right) \text { is monotone increasing }
$$

for arbitrary $\left(\left(x_{1}^{1},\left(x_{1}^{i}, x_{2}^{i}\right)\right) \in \mathbb{R}^{3}\right.$ such that $x_{1}^{1}>x_{1}^{i}$.

Once again, we shall deduce that the lower envelope $\bar{u}($.$) of the u_{i}$ 's is a continuous viscosity solution (10) from Prop. 3.1, by verifying hypothesis (C). Take any $z=\left(x^{1}, \ldots, x^{m+1}\right) \in\left(\mathbb{R}^{2} \times \ldots \mathbb{R}^{2}\right) \backslash \mathcal{T}$, any index set $I(z)$ (of cardinality $l>1$ ) such that the values $u_{i}(z), i \in I(z)$ coincide, and any convex combination $\left\{\lambda_{i}\right\}$ from $I(z)$. We may assume that index values have been re-ordered so that $I(z)=\{2, \ldots, l+1\}$. For $i=2, \ldots, l+1$, take any

$$
\tilde{p}^{i} \in \mathbb{R}^{n} \in \partial^{L} u_{i}(z)
$$

In consequence of the fact that $u_{i}\left(x^{1}, \ldots, x^{m+1}\right)$ depends only on $\left(x^{1}, x^{i}\right)$, we can write

$$
\tilde{p}^{i}:=\left(\left(p_{1}^{i, 1}, p_{2}^{i, 1}\right),(0,0), \ldots,(0,0),\left(p_{1}^{i}, p_{2}^{i}\right),(0,0), \ldots(0,0)\right) \in \partial^{L} u_{i}(z) .
$$

The possibly non-zero components $\left(p_{1}^{i, 1}, p_{2}^{i, 1}\right)$ and $\left(p_{1}^{i}, p_{2}^{i}\right)$ of $\tilde{p}^{i}$ appear at the first and $i$ 'th locations. Note that, by (12),

$$
p_{2}^{i, 1} \geq 0 \text { for } i=2, \ldots, m+1 .
$$

Verification of hypothesis $(\mathrm{C})$ requires us to show that $\eta\left(\lambda_{2}, \ldots, \lambda_{l+1}\right) \geq 0$, where

$$
\begin{aligned}
\eta\left(\lambda_{2}, \ldots, \lambda_{l+1}\right):= & \sum_{i=2}^{l+1} \lambda_{i} F^{21}\left(z, \tilde{p}_{i}\right)-F^{21}\left(z, \sum_{i=2}^{l+1} \lambda_{i} \tilde{p}_{i}\right) \\
& +\sum_{i=2}^{l+1} \lambda_{i} F^{22}\left(z, \tilde{p}_{i}\right)-F^{22}\left(z, \sum_{i=2}^{l+1} \lambda_{i} \tilde{p}_{i}\right) .
\end{aligned}
$$

Because $F^{21}(z,$.$) is linear, we have$

$$
\eta\left(\lambda_{2}, \ldots, \lambda_{l+1}\right):=\sum_{i=2}^{l+1} \lambda_{i} F^{22}\left(z, \tilde{p}_{i}\right)-F^{22}\left(z, \sum_{i=2}^{l+2} \lambda_{i} \tilde{p}_{i}\right)=c_{1}+c_{2}
$$

where

$$
c_{1}:=\sum_{i=2}^{l+1}\left(-\alpha \lambda_{i}\left|p_{2}^{i, 1}\right|+\alpha\left|\lambda_{i} p_{2}^{1}\right|\right) \text { and } c_{2}:=\sum_{i=2}^{l+1} \lambda_{i} f^{22}\left(p^{i}\right)-f^{22}\left(z, \sum_{i=2}^{l+1} \lambda_{i} p^{i}\right)
$$



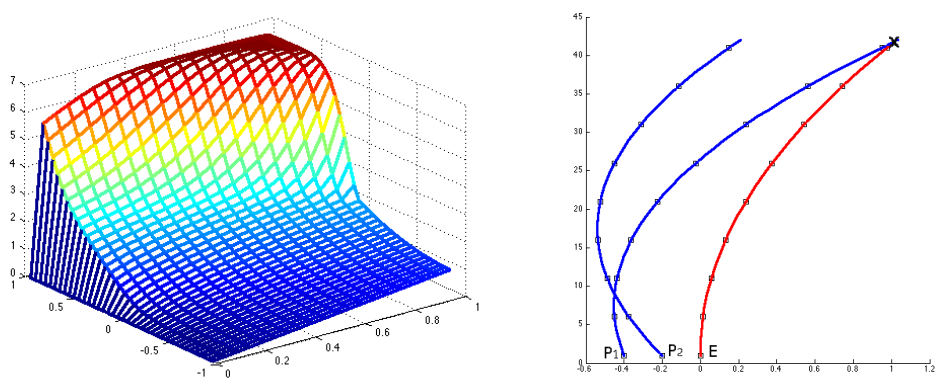

Fig. 1 Value function projected onto first two coordinates, in which case it coincides with the value function of a one-pursuer/one evader game w.r.t. reduced coordinates (left) and optimal trajectories for a two-pursuer/one evader game (first component over time). $X$ marks the point of capture (right).

in which

$$
f^{22}\left(\left(\left(p_{1}^{1}, p_{2}^{1}\right), \ldots,\left(p_{1}^{m+1}, p_{2}^{m+1}\right):=\sum_{i=2}^{m+1} \beta_{i} \times\left|p_{2}^{i}\right| .\right.\right.
$$

But $c_{1}=0$ since, by (13), the $p_{2}^{i, 1}$, s all have the same sign. Also, $c_{2} \geq 0$, by convexity of $f^{22}($.$) . We have confirmed \eta\left(\lambda_{2}, \ldots, \lambda_{l+1}\right) \geq 0$, and the proof of the proposition is complete.

For the special case when $m_{2}=2, d(x)=x, \alpha=1$ and $\beta_{1}=\beta_{2}=0.5$, (Figure 1, left) shows computations of the value function with respect to the reduced coordinates $\left(y^{1}, y^{2}\right)=\left(x_{1}^{1}-x_{1}^{2}, x_{2}^{1}-x_{2}^{2}\right)\left(y^{1}, y^{2}\right)=\left(x_{1}^{1}-x_{1}^{2}, x_{2}^{1}-x_{2}^{2}\right)$ in $\mathbb{R}^{2}$. Figure 1 (right) shows an example of the evolution of the positions of the players over time, with respect to the original coordinates. Capture occurs at the point marked $X$, when pursuer $P_{1}$ overtakes the evader, despite starting farther from the evader than pursuer $P_{2}$.

Verification of Claim: Consider first the case $z=z^{\prime}$. Fix $t>0$. We examine the optimal control problem of

$$
\left\{\begin{array}{l}
\text { minimize }-y_{1}(t) \text { subject to } \\
\left(\dot{y}_{1}(s), \dot{y}_{2}(s)\right)=\left(y_{2}(s),-d\left(y_{2}(s)\right)+a(s)\right), \text { a.e. } s \in[0, t], \\
a(s) \in[-1,+1], \text { a.e. } s \in[0, t],\left(y_{1}(0), y_{2}(0)\right)=\left(z_{1}, z_{2}\right) .
\end{array}\right.
$$

(Notice that the controlled differential equation in this problem is that governing the motion of the evader.) The data for the problem satisfy standard hypotheses for the existence of a minimizer $a^{*}($.$) on [0, t]$, with corresponding state trajectory $y^{*}($.$) (see, e.g. [24, Chap. 2]). We can establish, by means of a$ simple contradiction argument, that the nonsmooth Maximum Principle (see [24, Thm. 6.2.3]) applies in normal form. We deduce the existence of a costate arc $p()=.\left(p_{1}(),. p_{2}().\right)$ such that $p_{1}(.) \equiv+1$, and $p_{2}($.$) satisfies the differential$ 
equation and right endpoint boundary condition

$$
-\dot{p}_{2}(s)=+p_{1}(s)-\xi(s) p_{2}(s), \quad \text { for } s \in[0, t] \text { and } p_{2}(t)=0 .
$$

Here, $\xi_{i}($.$) is a Lipschitz continuous function satisfying \xi(s) \in \operatorname{co} \partial_{L} d_{1}\left(y_{2}^{*}(s)\right)$ a.e. in which $\partial_{L} d_{1}$ is the limiting subdifferential. The solution $p_{2}($.$) is strictly$ positive on $[0, t)$. From the 'maximization of the Hamiltonian'

$$
a^{*}(s)=\arg \max \left\{p_{2}(s) a: a \in[-1,+1]\right\}=+1,
$$

$a^{*}()=.\bar{a}($.$) on [0, t]$. We have shown that, for any $t \geq 0$ and initial condition $z, a()=.\bar{a}($.$) maximizes y_{1}(t)$. This confirms (11) when $z^{\prime}=z$.

We now show that (11) is true also when $z_{1}^{\prime}=z_{1}$ and $z_{2}^{\prime}>z_{2}^{\prime}$. In view of the preceding analysis, we can assume that $a()=.\bar{a}($.$) . Write \left(y_{1}(),. y_{2}().\right)$ and $\left(y_{1}^{\prime}(),. y_{2}^{\prime}().\right)$ for the solutions to the state equation, for the initial states $z=\left(z_{1}, z_{2}\right)$ and $z^{\prime}=\left(z_{1}^{\prime}, z_{2}^{\prime}\right)$ respectively. Take any time $\bar{t}>0$. By assumption $\dot{y}_{2}^{\prime}(0)>\dot{y}_{2}(0)$. So there are two cases to consider

(a): $\dot{y}_{2}^{\prime}(t)>\dot{y}_{2}(t)$ for all $t \geq 0$. In this case, since $y_{1}^{\prime}(0)-y_{1}(0)>0$, we have, as required,

$$
y_{1}^{\prime}(\bar{t})-y_{1}(\bar{t})=\left(y_{1}^{\prime}(0)-y_{1}(0)\right)+\int_{0}^{\bar{t}}\left(\dot{y}_{2}^{\prime}(t)-\dot{y}_{2}(t)\right) d t>0 .
$$

(b) There exists $t^{\prime} \in(0, \bar{t}]$ s.t. $\dot{y}_{2}^{\prime}(t)>\dot{y}_{2}(t)$ for $t \in\left[0, t^{\prime}\right)$ and $\dot{y}_{2}^{\prime}\left(t^{\prime}\right)=\dot{y}_{2}\left(t^{\prime}\right)$. In this case we show as, in the previous case, that $y_{1}^{\prime}\left(t^{\prime}\right)-y_{1}\left(t^{\prime}\right)>0$. We deduce from the uniqueness of solutions to the differential equation

$$
\dot{y}_{2}(t)=d\left(y_{2}\right)+1
$$

on $\left[t^{\prime}, \bar{t}\right]$, for fixed initial condition, that $y_{2}^{\prime}(t)=y_{2}(t)$ for $t \in\left[t^{\prime}, \bar{t}\right]$. Hence, again, we arrive at the desired relation:

$$
y_{1}^{\prime}(\bar{t})-y_{1}(\bar{t})=\left(y_{1}^{\prime}\left(t^{\prime}\right)-y_{1}\left(t^{\prime}\right)\right)+\int_{t^{\prime}}^{\bar{t}}\left(\dot{y}_{2}^{\prime}(t)-\dot{y}_{2}(t)\right) d t>0 .
$$

\subsection{A Pursuit/Evasion Game With No Decomposition}

We now provide a simple example illustrating that, for a multiple pursuers/single evader game, with target a union of target subsets, each associated with the evader and just one of the pursuers, may fail to have a decomposition. In this example, it is possible to derive formulae for the value functions involved, and to test the conditions for decomposition directly.

We denote by $\left(P^{3}\right)$ the special case of $(P)$ in which $m_{1}=1, m_{2}=2$ and $n=1$.

$$
f_{1}\left(x_{1}, a\right)=a, f_{2}\left(x_{2}, b_{1}\right)=b_{1} \text { and } f_{3}\left(x_{3}, b_{2}\right)=b_{2} .
$$


The controls actions of the players are constrained as follows:

$$
\begin{gathered}
a \in A:=[-\alpha,+\alpha], \\
b_{1} \in B_{1}:=[-1,+1] \text { and } b_{2} \in B_{2}:=[-1,+1],
\end{gathered}
$$

for some $\alpha \in(0,1)$. We take the target to be

$$
\begin{gathered}
\mathcal{T}=\mathcal{T}_{2} \cup \mathcal{T}_{3}, \quad \text { where } \\
\mathcal{T}_{2}=\left\{\left(x_{1}, x_{2}, x_{3}\right): x_{1}=x_{2}\right\}, \mathcal{T}_{3}=\left\{\left(x_{1}, x_{2}, x_{3}\right): x_{1}=x_{3}\right\} .
\end{gathered}
$$

(In this version of the game, two pursuers chase a single evader in $1 D$ space. The game terminates when either pursuer meets the evader.) Denote by $\left(P_{2}^{3}\right)$ and $\left(P_{3}^{3}\right)$ the modified games in which the target $\mathcal{T}$ is replaced by the subsets $\mathcal{T}_{2}$ and $\mathcal{T}_{3}$ respectively. The HJI equation is

$$
F^{3}\left(D_{x_{1}} u, D_{x_{2}} u, D_{x_{3}} u\right)=0,
$$

in which $F^{3}\left(p_{1}, p_{2}, p_{3}\right)=\left|p_{2}\right|+\left|p_{3}\right|-\alpha\left|p_{1}\right|-1$.

Optimal strategies for both games $\left(P_{2}^{3}\right)$ and $\left(P_{3}^{3}\right)$ are: the evader moves away from the pursuer, and the pursuer moves towards the evader, as quickly as possible. A simple calculation based on these observations yields upper values for $\left(P_{2}^{3}\right)$ and $\left(P_{3}^{3}\right)$, namely:

$$
\begin{aligned}
& u_{2}\left(x_{1}, x_{2}, x_{3}\right)=(1-\alpha)^{-1}\left|x_{2}-x_{1}\right|, \\
& u_{3}\left(x_{1}, x_{2}, x_{3}\right)=(1-\alpha)^{-1}\left|x_{3}-x_{1}\right|,
\end{aligned}
$$

for all $x=\left(x_{1}, x_{2}, x_{3}\right) \in \mathbb{R}^{3}$. Define $\bar{u}():. \mathbb{R}^{3} \rightarrow \mathbb{R}$ to be

$$
\bar{u}(x)=\min \left\{u_{1}(x), u_{2}(x)\right\} \text { for } x \in \mathbb{R}^{3} .
$$

Proposition $4.3 \bar{u}($.$) is not a continuous viscosity solution for (14) on \mathbb{R}^{3} \backslash \mathcal{T}$.

Since the upper value for $\left(P^{3}\right)$ is a viscosity solution on $\mathbb{R}^{3} \backslash \mathcal{T}$, vanishing on $\mathcal{T}$, we may conclude that $\bar{u}($.$) is not the value function for \left(P^{3}\right)$.

Proof Take any $z>0$ and let $\bar{x}=(0, z,-z)$. Then $\bar{x} \in \mathbb{R}^{3} \backslash\left(\mathcal{T}_{2} \cup \mathcal{T}_{3}\right)$. Also, $u_{2}(\bar{x})=u_{3}(\bar{x}), u_{2}($.$) and u_{3}($.$) are continuously differentiable at \bar{x}$. From the formulae for the value functions we have

$$
\begin{aligned}
& D u_{2}(\bar{z})=\left(-(1-\alpha)^{-1},(1-\alpha)^{-1}, 0\right), \text { and } \\
& D u_{3}(\bar{z})=\left((1-\alpha)^{-1}, 0,-(1-\alpha)^{-1}\right) .
\end{aligned}
$$

Then, for any $\lambda \in(0,1)$,

$$
\begin{aligned}
F^{3}\left(\lambda D_{x} u_{2}(\bar{x})+(1-\lambda) D_{x} u_{3}(\bar{x})\right) & =\frac{\lambda}{(1-\beta)}+\frac{1-\lambda}{(1-\beta)} \\
-\frac{\beta}{(1-\beta)}(-\lambda+(1-\lambda))-1 & =\frac{2 \lambda \beta}{(1-\beta)}>0 .
\end{aligned}
$$


So condition $(E)$ is violated. But according to Prop 3.1, $(E)$ is a necessary condition that the lower envelope $\bar{u}($.$) is a continuous viscosity subsolution. It$ follows that $\bar{u}($.$) cannot be a viscosity solution to (14).$

The true value function $u($.$) for \left(P^{3}\right)$ is expressed in terms of the subset:

$$
\begin{aligned}
\mathcal{D}= & \left\{\left(x_{1}, x_{2}, x_{3}\right) \in \mathbb{R}^{3}: \operatorname{sgn}\left\{x_{2}-x_{1}\right\}=-\operatorname{sgn}\left\{x_{3}-x_{1}\right\}\right. \\
& \text { and } \left.\frac{1-\alpha}{1+\alpha}\left|x_{3}-x_{1}\right|<\left|x_{2}-x_{1}\right|<\frac{1+\alpha}{1-\alpha}\left|x_{3}-x_{1}\right|\right\} .
\end{aligned}
$$

It is

$$
u\left(x_{1}, x_{2}, x_{3}\right)=\left\{\begin{array}{c}
\frac{1}{1-\alpha} \min \left\{\left|x_{2}-x_{1}\right|,\left|x_{3}-x_{1}\right|\right\} \\
\text { for }\left(x_{1}, x_{2}, x_{3}\right) \in \mathbb{R}^{3} \backslash \mathcal{D} \\
\frac{1}{2}\left(\left|x_{2}-x_{1}\right|+\left|x_{3}-x_{1}\right|\right) \\
\text { for }\left(x_{1}, x_{2}, x_{3}\right) \in \mathcal{D}
\end{array}\right.
$$

We see that $u($.$) coincides with \min \left\{u_{1}(x), u_{2}(x)\right\}$, for $x \in \mathbb{R}^{3} \backslash \mathcal{D}$. But

$$
u(x)<\min \left\{u_{1}(x), u_{2}(x)\right\}, \text { for } x \in \mathcal{D} .
$$

(The value function is constructed according to the heuristic: 'each of the pursuers always travels at maximum speed towards the evader. If both pursuers are on the same side of the evader, the evader travels at maximum speed in the opposite direction until the evader is hit. If, on the other hand, the evader is between the two pursuers, the evader travels at maximum speed away from the closest pursuer until the two pursuers are equidistant. The evader then stops until the evader is reached by one of the pursuers'. A check is then carried out that the value function is a continuous viscosity solution of (5), has a continuous extension to $\mathcal{T}$ on which it vanishes, and which is therefore the upper value of the game.)

\section{Flow Control}

A surge tank is a buffering device, used to smooth the flow of a fluid between two chemical reactors. Let $x_{1}$ be the height of the fluid in the surge tank. Then $x_{1}$ is related to inflow rate $b$ and the outflow rate $a$ according to

$$
d^{2} x_{1} / d t^{2}=b-a .
$$

We treat $b$ and $a$ as a disturbance signal and a control signal respectively. In normalized units, the requirement that the surge tank must not overflow or empty is captured by the constraint:

$$
-1 \leq x_{1} \leq+1
$$

There is also a constraint on the maximum outflow rate which, if excessive, can give rise to turbulent flow/sediment displacement which is disruptive for the chemical processing:

$$
-1 \leq a \leq+1
$$


The control design problem is to find a feedback control

$$
a=\chi\left(x_{1}, \dot{x}_{1}\right),
$$

compatible with the constraint (15), which counters the tendency of the surge tank to either empty or overflow.

For surge tanks systems, proportional + integral (PI) controllers can fail to satisfy performance requirements, because control action is insufficiently aggressive when the height almost violates the constraint (5) and a number of non-classical controllers have been proposed, including a model predictive controller [5], a variable structure controller [17], [20] and a games-theoretic controller [10]. Following [10], we formulate the control design problem as an example of the exit problem of the introduction, when the 2 dimension state vector is $x^{T}=\left(x_{1}, x_{2}:=\dot{x}_{1}\right)$ (the fluid volume and the rate of change of fluid volume). The players' actions are constrained by $a \in \Omega_{1}$ and $b \in \Omega_{1}$ where

$$
\left.\Omega_{1}:=[-1,+1] \text { and } \Omega_{2}:=\right]-\infty,+\infty[
$$

and the dynamic constraint is

$$
\dot{x}=f(x, a, b) \quad \text { a.e. }
$$

in which $f(x, a, b)=H x+g(-a+b)$. Here, the matrix $H$ and vector $g$ are

$$
H=\left[\begin{array}{ll}
0 & 1 \\
0 & 0
\end{array}\right] \quad g=\left[\begin{array}{l}
0 \\
1
\end{array}\right]
$$

The 'safe' region of the state space $\mathbb{R}^{2}$ is $[-1,+1] \times \mathbb{R}$ which can be expressed as the complement of the target:

$$
\mathcal{T}=\mathcal{T}_{1} \cup \mathcal{T}_{2}
$$

where the two disjount sets $\mathcal{T}_{1}$ and $\mathcal{T}_{2}$ are

$$
\mathcal{T}_{1}=\left[+1,+\infty\left[\times \mathbb{R} \text { and } \mathcal{T}_{2}=[-\infty,-1) \times \mathbb{R} .\right.\right.
$$

By choosing the payoff to be

$$
J\left(x_{0}, a(.), b(.)\right)=\frac{1}{2} \int_{0}^{\tau} b^{2}(t) d t
$$

in which, as before, $\tau$ is the first exit time into $\mathcal{T}$, we can be sure that the best closed loop policy for the $a$-player will maximize the minimum energy the $b$ player must expend, to escape into $\mathcal{T}$.

Now consider a decomposition of the value function $u($.$) for this exit problem,$ in terms of the value functions $u_{j}(),. j=1,2$, for two modifications $\left(P_{x_{0}}^{j}\right)$, $j=1,2$, of the exit problem, which result when we replace $\mathcal{T}$ by $\mathcal{T}_{j}, j=1,2$. Consider $\left(P_{x_{0}}^{1}\right)$. The target set is $\mathcal{T}=[+1, \infty[$ ('the surge tank overflows'). Here, the maximizing closed loop policy for the $a$-player is

$$
a^{*}(.) \equiv+1
$$


(i.e. the maximizing mapping $\phi^{*}():. \mathcal{A} \rightarrow \mathcal{B}$ is $\phi(a()).(t)=+1$ for all $b(.) \in \mathcal{B}$.) This is because, for any other closed loop policy for the $a$-player, the exit time must be reduced for any open-loop policy by the $b$-player, since the rate of outflow will be increased. Since the cost integrand is non-negative, this means the exit payoff is decreased. Likewise, for problem $\left(P_{x_{0}}^{2}\right)$, the maximizing closed loop policy for the $a$-player is

$$
a^{*}(.):=-1 .
$$

Because the maximizing closed loop policies for the $a$-player are constant, the exit problems $\left(P_{x_{0}}^{1}\right)$ and $\left(P_{x_{0}}^{1}\right)$ take the form of optimal control problems, in which we take the dynamic constraints are $\dot{x}=\tilde{f}_{1}(x, b)=f(x,+1, b)$ and $\dot{x}=\tilde{f}_{2}(x, b)=f(x,-1, b)$, respectively:

$$
\left(P_{x_{0}}^{1}\right)\left\{\begin{array}{l}
\text { minimize } \frac{1}{2} \int_{0}^{\tau}|v|^{2} d t \\
\text { over } \left.\tau \geq 0 \text { and } v \in L^{2}[0, \tau] ; R\right) \text { satisfying } \\
\dot{x}=H x+b(-1+v) \text { a.e. } t \in[0, \tau] \\
x(0)=x_{0}, x(\tau) \in\{+1\} \times R .
\end{array}\right.
$$

and

$$
\left(P_{x_{0}}^{2}\right)\left\{\begin{array}{l}
\operatorname{minimize} \frac{1}{2} \int_{0}^{\tau}|v|^{2} d t \\
\text { over } \left.\tau \geq 0 \text { and } v \in L^{2}[0, \tau] ; R\right) \text { satisfying } \\
\dot{x}=H x+b(+1+v) \text { a.e. } t \in[0, \tau] \\
x(0)=x_{0}, x(\tau) \in\{-1\} \times R
\end{array}\right.
$$

We call the minimum costs these two optimal control problems, regarded as functions of the initial state, their value functions.

Solution of the Optimal Control Problems. For each $x \in x_{0} \in \mathbb{R}^{2} \backslash \mathcal{T}_{j}, j=$ $1,2,\left(P^{j}, x_{0}\right)$ has a minimizer, as may be deduced from standard criteria for existence of minimizers (see, e.g. [24, Chap. 2]). Application of the free time Maximum Principle and analysis of the extremality conditions establishes that, for $j=1,2$, and given initial state $x_{0} \in \mathbb{R}^{2} \backslash \mathcal{T}_{j}$, there is a unique extremal. Since $\left(P_{x_{0}}^{j}\right)$ has a minimizer, the extremal is this minimizer. Formulae can then be derived for the minimizing control and the value function after a change of coordinates. The change of coordinates involves the mappings $\eta^{1}$ : $\left(0, \infty\left[\times\left(0, \infty\left[\rightarrow \mathbb{R}^{2}\right.\right.\right.\right.$ and $\eta^{2}:\left(0, \infty\left[\times\left(0, \infty\left[\rightarrow \mathbb{R}^{2}:\right.\right.\right.\right.$

$$
\begin{aligned}
& \eta^{1}(q, \tau)=\left(1-\frac{1}{2} \tau^{2}+\frac{1}{6} q \tau^{3}, \tau-\frac{1}{2} q \tau^{2}\right), \\
& \eta^{2}(r, \sigma)=\left(-1+\frac{1}{2} \sigma^{2}-\frac{1}{6} r \sigma^{3},-\sigma+\frac{1}{2} r \sigma^{2}\right) .
\end{aligned}
$$

and the subsets

$$
\begin{aligned}
& D_{1}:=\left\{\left(x_{1}, x_{2}\right) \in \mathbb{R}^{2} \backslash \mathcal{T}_{1}: x_{1}<1-\frac{1}{2}\left(x_{2} \vee 0\right)^{2}\right\}, \\
& D_{2}:=\left\{\left(x_{1}, x_{2}\right) \in \mathbb{R}^{2} \backslash \mathcal{T}_{2}: x_{1}>-1+\frac{1}{2}\left(x_{2} \wedge 0\right)^{2}\right\} .
\end{aligned}
$$


Then, for some open sets $\mathcal{O}^{j}, j=1,2, \eta^{j}($.$) is one-to-one on \mathcal{O}^{j}$ and

$$
\eta^{j}\left(\mathcal{O}^{j}\right)=D_{j}
$$

It can be shown that the support set of $u_{j}($.$) , i.e. the subset of \mathbb{R}^{2} \backslash \mathcal{T}$ on which $u_{j}($.$) is strictly positive, is precisely D_{j}$, for $j=1,2$. Furthermore, for any initial state $x \in\left(\mathbb{R}^{2} \backslash \mathcal{T}_{j}\right) \cap D_{j}$, the value function is continuously differentiable on a neighborhood of $x$, and the value function, value function gradient, first exit time and optimal control for problem $\left(P_{x}^{1}\right)$ are given by

$$
\left.\begin{array}{l}
u_{1}(x)=\frac{1}{6} q^{2} \tau^{3} \\
D u_{1}(x)=(-q,-q \tau) \\
\text { first exit time }=\tau \\
b^{*}(t)=q(\tau-t), \quad \text { for a.e. } t \in[0, \tau]
\end{array}\right\}
$$

in which $(q, \tau)=\left(\eta^{1}\right)^{-1}(x)$, and

$$
\left.\begin{array}{l}
u_{2}(x)=\frac{1}{6} r^{2} \sigma^{3} \\
D u_{2}(x)=(r, r \sigma) \\
\text { first exit time }=\sigma \\
b^{*}(t)=-r(\sigma-t), \quad \text { for a.e. } t \in[0, \sigma]
\end{array}\right\}
$$

in which $(r, \sigma)=\left(\eta^{2}\right)^{-1}(x)$.

It can be also shown that the set of points on which $u_{1}($.$) and u_{2}($.$) coincide$

$$
\Sigma:=\left\{\left(x_{1}, x_{2}\right) \in \mathbb{R}^{2} \backslash \mathcal{T}: u_{1}(x)=u_{2}(x)\right\}
$$

is a subset of the open set $D_{1} \cap D_{2}$. Also, for an arbitrary point $x \in \Sigma, u_{1}($.) and $u_{2}($.$) are continuously differentiable on a neighborhood of x$.

We now address the question of whether the value function of $\left(P_{x_{0}}\right)$ can be calculated as the lower envelope of the value functions $u_{1}($.$) and u_{2}($.$) for the$ two control problems. It hinges on the question of whether the the lower envelope is a viscosity equation of the Hamilton Jacobi equation. This is because, as can be shown, the value function is the unique viscosity solution that is continuous on the $\mathbb{R}^{2} \backslash \mathcal{T}$, and has a continuous extension to the "exit boundary' $^{\prime}\{+1\} \times[0,+\infty[) \cup(\{-1\} \times[-\infty, 0[)$ on which it vanishes. Since the lower envelope, also, has a continuous extension to the exit boundary, on which it vanishes, the lower envelope must be the upper value for the game, if the lower envelope is a viscosity solution.

In this example, the left side of the the HJI, $F(x, u(x), D u(x))=0$ takes the form:

$$
F(x, u, p)=p_{1} x_{2}-\left|p_{2}\right|+\frac{1}{2}\left|p_{2}\right|^{2}-1 .
$$

The Isaac's condition (1) is satisfied. Notice that $F(x, u,$.$) fails to be convex$ because of the presence of the $-\left|p_{2}\right|$ term in this formula. However the theory of Section 2 yields: 
Proposition 5.1 Let $u_{j}($.$) be the value functions of the optimal control prob-$ lems $\left(P_{x_{0}}^{j}\right)$ associated with the target sets $\mathcal{T}_{j}, j=1,2$. Then the function

$$
\bar{u}(x):=\min \left\{u_{1}(x), u_{2}(x)\right\}
$$

is a viscosity solution of the Hamilton Jacobi equation on $\mathbb{R}^{2} \backslash \mathcal{T}$, where $F(., .,$. is the function (18). $\bar{u}($.$) therefore coincides with the upper value u($.$) on \mathbb{R}^{2} \backslash \mathcal{T}$.

Proof Since $F(., .,$.$) is continuous, it will follow from Prop. 3.1$ that $\bar{u}($.$) is a$ viscosity solution on $\mathbb{R}^{2} \backslash \mathcal{T}$ if we can demonstrate that hypothesis $(C)$ of the proposition is satisfied. We now show this. Take any $\left(x_{1}, x_{2}\right) \in \Sigma$, the set on which $u_{1}($.$) and u_{2}($.$) coincide with \bar{u}($.$) . Both u_{1}($.$) and u_{j}($.$) are continuously$ differentiable on a neighborhood of $x$. So we must check the condition

$$
\left.F\left(x, \bar{u}(x), \alpha p_{1}+(1-\alpha) p_{2}\right)\right) \leq \alpha F\left(x, \bar{u}(x), p_{1}\right)+\alpha F\left(x, \bar{u}(x), p_{2}\right),
$$

for $p_{1}=D u_{1}(x)$ and $p_{2}=D u_{2}(x)$.

We know from (16) and (17) that there exist $\tau, q \in(0,+\infty[$, and $\sigma, r \in(0,+\infty[$ such that $(\tau, q) \in \eta^{1}(x)$ and $(\sigma, r) \in \eta^{2}(x)$, and

$$
\begin{aligned}
& \tau q=(\sigma / \tau)^{\frac{1}{2}} \sigma r \\
& \left(1-\frac{1}{2} \tau q\right)=-(\sigma / \tau)\left(1-\frac{1}{2} r \sigma\right) \\
& D u_{1}(x)=\tau q \text { and } D u_{2}(x)=\sigma r .
\end{aligned}
$$

Condition $(\mathrm{C})$ requires that

$$
\min \{\xi(\alpha): \alpha \in[0,1]\} \geq 0
$$

where

$$
\begin{array}{r}
\xi(\alpha):=\alpha F\left(x, \bar{u}, D u_{1}(x)\right)+(1-\alpha) F\left(x, \bar{u}, D u_{2}(x)\right)- \\
F\left(x, \bar{u}, \alpha D u_{1}(x)+(1-\alpha) u_{2}(x)\right)
\end{array}
$$

But, by (21), we have, for any $\alpha \in[0,1]$,

$$
\begin{aligned}
\xi(\alpha)=\alpha(-\tau q+ & \left.\frac{1}{2}(\tau q)^{2}\right)+(1-\alpha)\left(-\sigma r+\frac{1}{2}(\sigma r)^{2}\right) \\
& +\left(-|\alpha \tau q-(1-\alpha) \sigma r|+\frac{1}{2}|\alpha \tau q-(1-\alpha) \sigma r|^{2}\right) .
\end{aligned}
$$

Now define

$$
\bar{\alpha}:=\frac{\sigma r}{\tau q+\sigma r}(\in[0,1]) .
$$

We observe that the restrictions of $\xi($.$) to the intervals [0, \bar{\alpha}]$ and $[\bar{\alpha}, 1]$ are both concave functions. It follows that the minimum of $\xi($.$) must be achieved$ at $\alpha=0,1$ or $\bar{\alpha}$. If the minimum is achieved at 0 or at 1 then consequently $\min \{\xi(\alpha): \alpha \in[0,1]\}=0$, which implies (5). It remains to consider the case 


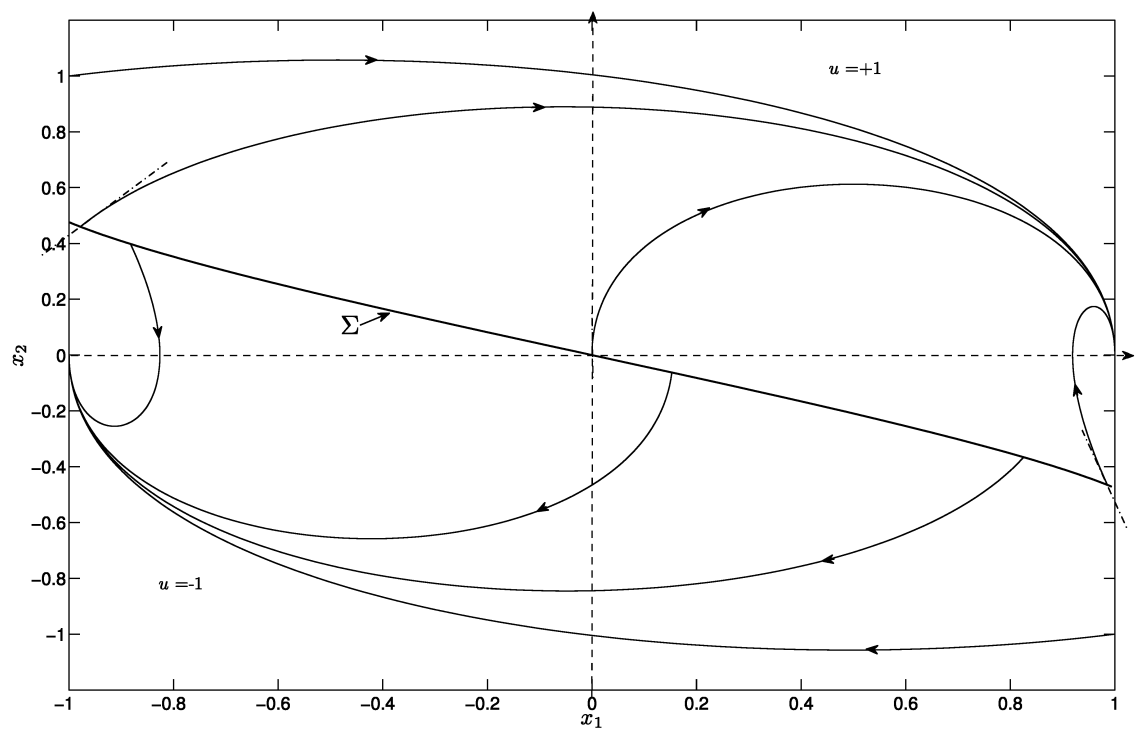

Fig. 2 Optimal trajectories of the flow control problem

when the minimum is achieved at $\alpha=\bar{\alpha}$. Since $\alpha \tau q-(1-\alpha) \sigma r=0$ when $\alpha=\bar{\alpha}$ we have

$$
\begin{aligned}
\min \{\xi(\alpha): \alpha \in[0,1]\} & =\xi(\bar{\alpha}) \\
& =\frac{\sigma r}{\tau q+\sigma r}\left(-\tau q+\frac{1}{2}(\tau q)^{2}\right)+\frac{\tau q}{\tau q+\sigma r}\left(-\sigma r+\frac{1}{2}(\sigma r)^{2}\right) \\
& =\frac{(\tau q)(\sigma r)}{\tau q+\tau q)(\sigma r}\left(\left(\frac{1}{2} \tau q-1\right)+\frac{1}{2} \sigma r\right) \\
& =\frac{(\tau q)(\sigma r)}{\tau q+\tau q)(\sigma r}\left(\frac{1}{2} \tau q-1\right)\left(1-\frac{\tau}{\sigma}\right) .
\end{aligned}
$$

(We have used (20) to derive the last line.)

But, by (20), either $\frac{1}{2} \tau q \geq 1$ and $\frac{1}{2} \sigma r \leq 1$ or vice versa. If the first alternative is true then, by $(19),(\tau / \sigma) \leq 1$, both terms in the product $\left(\frac{1}{2} \tau q-1\right)\left(1-\frac{\tau}{\sigma}\right)$ are positive, and (5) follows from the preceeding relation. If, on the other hand, the second alternative is true then $(\sigma / \tau) \leq 1$ and both terms in the product $\left(\frac{1}{2} \tau q-1\right)\left(1-\frac{\tau}{\sigma}\right)$ are negative. We see that (5) follows, once again, from the previous relation. Condition $(\mathrm{C})$ is confirmed.

Fig. 2 shows the set $\Sigma$ on which $u_{1}($.$) and u_{2}($.$) coincide, and some trajectories$ achieving the upper value of the game.

Remark It was shown in [10], by means of a direct analysis making heavy use of the specific structure of the flow control problem, that the upper value of the flow control problem can be calculated as the lower envelope of the value functions for the control problems $\left(P_{x_{0}}^{1}\right)$ and $\left(P_{x_{0}}^{1}\right)$. This analysis involved 
checking monotonicity properties, 'non-intersecting extremal' properties and special arguments to exclude pathological behavior on the set $\Sigma$, and was very complicated. Here, we achieve the same results, but by means of a much simpler analysis, which involves merely checking the validity of the restricted convexity condition (C) in Prop. 3.1.

\section{Perspectives}

The distinguishing feature of many modern-day application areas of differential games, such as collision avoidance, air traffic control and nonlinear robust control, is the high dimension of the state variable. For applications of this nature, techniques are required to reduce the complexity of such games and thereby rendering their solutions computable. The techniques presented in this paper, applicable in situations where we can exploit special structure of the target set, adds to the library of available methods. It should be seen as part of a broader program of research into complexity reduction for differential games. This paper leaves open a number of questions. The theoretical core of this paper are sets of criteria in zero sum games, which permit the construction of the value function as the lower envelope of value functions for simpler differential games. These criteria can no doubt be replaced by less restrictive ones. In particular, we might hope to relax the Lipschitz continuity conditions on the constituent value functions under which this representation is achievable. A full characterization of the classes of differential games permitting 'decompositon by target sub-sets' is so far lacking. Finally, it would be desirable for other possible areas of application to be explored, for example in mathematical economics and operations research.

\section{Conclusions}

In pursuit evasion games and in other zero-sum differential games which terminate when the state reaches a target set, the target can often be described as a union of sub-targets, each of which describes just one of possible mode of termination. In this paper we have exploited this structure to decompose such a game into a family of simpler games, each member of which involves a target subset, in place of the original target set. Decomposition via target subsets is not a universal approach to reducing the complexity of zero-sum differential games. But the examples presented in this paper, in pursuit/evasion games and flow control in the presence of disturbances, illustrate the effectiveness of this approach in some significant cases. The length of the paper arises from a desire to demonstrate the usefulness of the proposed technique for a range of applications.

Acknowledgements: This this research was supported by the European Union under the 7th Framework Programme FP7-PEOPLE-2010-ITN SADCO, "Sensitivity Analysis for Deterministic Controller Design". 


\section{References}

1. Bardi M., Capuzzo-Dolcetta I.:Optimal Control and Viscosity Solutions of HamiltonJacobi-Bellman Equations. Birkhäuser, Boston (1997)

2. Bardi M., Raghavan T.E.S., Parthasarathy T.: Stochastic and Differential Games: Theory and Numerical Methods. Birkhäuser, Boston, (1999)

3. Barles G.: Solutions de viscositè des equations d'Hamilton-Jacobi. Springer-Verlag (1998)

4. Cacace S., Cristiani E., Falcone M., Picarelli A.: A Patchy Dynamic Programming Scheme for a Class of Hamilton-Jacobi-Bellman Equations. SIAM J. Sci. Comput. 34(5), A2625-A2649 (2012)

5. Campo P. J.: Model Predictive Optimal Averaging Level Control. AIChE J. 35(4), 579-591 (1989)

6. Cannarsa P., Sinestrari C.: Semiconcave functions, Hamilton-Jacobi equations, and optimal control. Birkhäuser, Boston (2004)

7. Chodun W.: Differential games of evasion with many pursuers. J. Math. Anal. Appl. 142(2), 370-389 (1989)

8. Elliott R.J., Kalton N.J.: Values in differential games. Bull. Amer. Math. Soc. 78(3), 427-431 (1972)

9. Falcone M.: Numerical Mehtods for Differential Games via PDEs. Int. Game Theor. Rev. 8(2), 231-272 (2006)

10. Falugi P., Kountouriotis C., Vinter R.B.: Differential Games controllers that confine a system to a safe region in the state space, with applications to surge tank control. IEEE T. Automat. Control 57(11), 2778-2788 (2012)

11. Festa A.: Reconstuction of Independent Sub-Domains for a class of Hamilton Jacobi Equations and its Application to Parallel Calculus. ESAIM M2AN doi:10.1051/m2an/201507 (2015)

12. Festa A., Vinter R.B.: A decomposition technique for pursuit evasion games with many pursuers. In: Proc. 52nd IEEE Control and Decision Conference (CDC) pp. 5797-5802 (2012)

13. Friedman A.: Differential Games. John Wiley \& Sons, New York, USA (1971)

14. Ibragimov G.I.: Optimal pursuit of an evader by countably many pursuers. Differ. Equ. 41(5), 627-635 (2005)

15. Isaacs R.: Differential Games. John Wiley \& Sons, New York, USA (1965)

16. Ivanov R.P., Ledyaev Y. S.: Time optimality for the pursuit of several objects with simple motion in a differential game. Trudy Mat. Inst. Steklov. 158, 87-97 (1981)

17. Kantor J.C.: Non-linear sliding mode controller and objective function for surge tanks. Int. J. Control 50, 2025-2047 (1989)

18. N.N. Krasovskii and A.I. Subbotin, Game-Theoretical Control Problems, Springer, New York, 1988.

19. Ledyaev Y.S., Treiman J.S.: Sub-and supergradients of envelopes, semicontinuous closures, and limits of sequences of functions. Russ. Math. Surv. 67(2), 345-373 (2012)

20. McDonald K. A., McAvoy T. J.: Optimal Averaging Level Control. AIChE J. 32, 75-86 (1986)

21. Pshenichnii B.N.: Simple pursuit by several objects. Cybern. Syst. Anal. 12(3), 484-485 (1976)

22. Subbotin A. I.: Generalized Solutions of First-Order PDEs. Birkhäuser, Boston (1995)

23. Vidal R., Shakernia O., Kim J., Shim H., Sastry S.: Probabilistic Pursuit-Evasion Games: Theory, Implementation, and Experimental Evaluation. IEEE T. Robotic. Autom. 18(5) 662-669 (2002)

24. Vinter R.: Optimal Control, Birkhäuser, Boston (2000) 